\title{
Predicting the Quench Behavior of the LHC Dipoles During Commissioning
}

\author{
C. Lorin, A. Siemko, E. Todesco, and A. Verweij
}

\begin{abstract}
The LHC hardware commissioning has shown a considerable difference of performance between the three dipole manufacturers (Firm1, Firm2 and Firm3). More than $90 \%$ of the quenches occurred in the dipoles made by Firm3, less than $10 \%$ in Firm2, and no one in Firm1. In this paper we propose a Monte-Carlo method based on the quench performance data of individual magnets that accounts for this behavior. The model relies on the data of the virgin training and on correlations with quench after a thermal cycle as measured on a sample of magnets. This model is used to derive estimates of the training behavior in the machine. A comparison with data gathered during the hardware commissioning shows that the model works well for low fields, and that, starting from fields corresponding to an energy of about 6.3 TeV, a slower training in the magnets of one manufacturer is observed.
\end{abstract}

Index Terms-Current density, stress, superconducting magnets.

\section{INTRODUCTION}

A FEW MONTHS ago, a New York Times article [1] reported that the LHC dipoles unexpectedly behaved below specifications during first tests in 2008. This shows the interest that this pretty technical topic has risen not only in the scientific community, but well beyond, up to the headlines of newspapers. Even though the lower performance of some magnets could reduce the energy of the LHC by only a few percent, this effect is relevant for the community of accelerator magnet builders, and deserves an in-depth analysis.

The quench behavior of superconducting magnets is one of the most fascinating and elusive topics of accelerator technology, lying in the no-man-land between superconductivity, mechanical engineering and electrical engineering [2], [3]. When a magnet is powered for the first time, it does not usually reach the current corresponding to the superconducting properties measured on a short sample of the cable (short sample limit): it has a first quench at 70-90\% of this limit (see Fig. 1). During successive quenches, the current level increases, i.e. the magnet trains. It is general belief that this is due to the Lorentz forces, which successively set the coil in a more stable position [2]-[6]. If the magnet is well conceived and built, it rapidly approaches the short sample limit after a few quenches. The memory of this training is usually partially kept after a warm-up and successive cool-down of the magnet (see Fig. 1).

Manuscript received October 29, 2009. First published March 25, 2010; current version published May 28, 2010.

The authors are with CERN-Technology Department, Geneva 23, $1211 \mathrm{CH}$, Switzerland (e-mail: ezio.todesco@cern.ch).

Color versions of one or more of the figures in this paper are available online at http://ieeexplore.ieee.org.

Digital Object Identifier 10.1109/TASC.2010.2043076

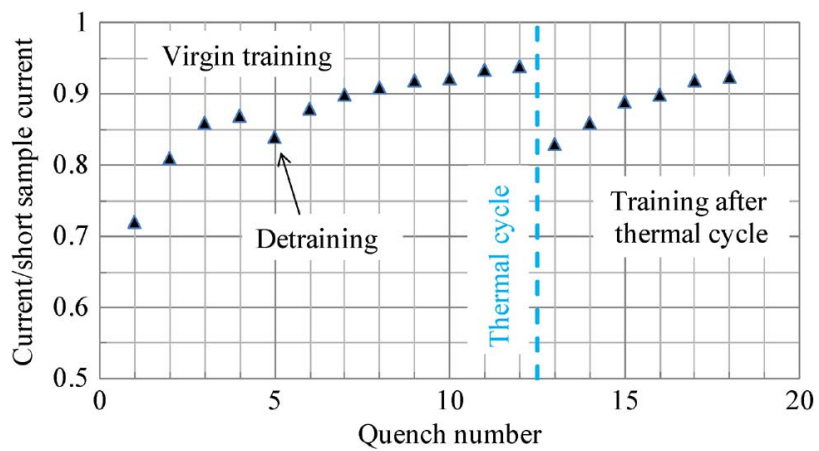

Fig. 1. Training of a magnet and training retention after thermal cycle.

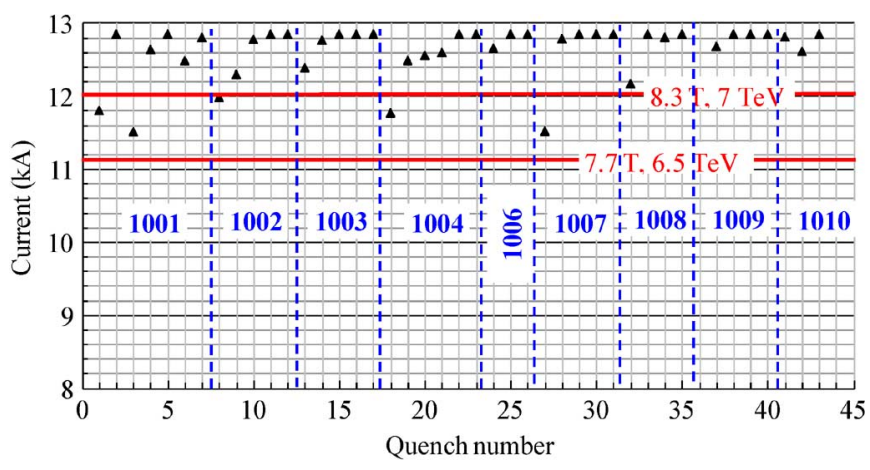

Fig. 2. Quench data of the first 9 LHC dipoles built by Firm1, in virgin conditions.

A thermal cycle will always occur before installation in the machine after testing, and also, during the accelerator lifetime, to allow intervention for maintenance or repair.

A disturbing feature of training is that it varies widely, even between magnets built with the same components and procedures (see Fig. 2). This is why it is difficult to pin point the dependence of the quench behavior on the magnet features during a model or prototyping phase: one assumes correlations between design changes and quenches which are only an effect of the intrinsic variability of the quench behavior.

In the LHC case, all magnets were built with the same recipe by three manufacturers, labeled Firm1, 2 and 3, with a few minor differences in tooling and procedures, and they have all been tested individually before installation. The commissioning of the LHC magnets [7], [8] offers a unique chance of having a wide phenomenology of the quench behavior on a set of 1200 dipoles. Here, we give an overview of the data gathered during the test campaign of all LHC dipoles. We propose a novel type of plot, cumulating the performance of individual magnets, showing a remarkable regularity in the quench behavior of 


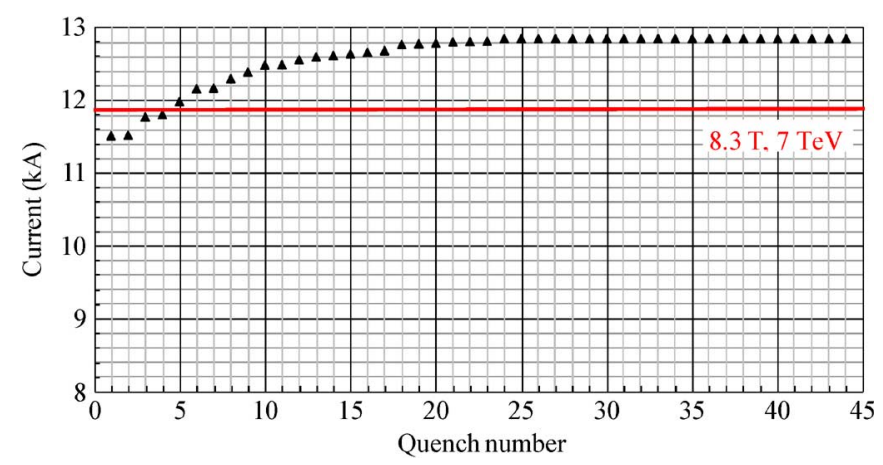

Fig. 3. Same data of Fig. 2, reordering the quenches by increasing current.

a large set of identical magnets. This plot also suggests a phenomenological fit to describe magnet training, based on an exponential fit. The unexpected lower training retention of Firm3 magnets is then described, and partially justified through a Monte-Carlo method.

\section{Quench Performance During Test}

\section{A. Main Features of the Test}

All the LHC dipoles were tested at the surface during the 5 -year-long production [9]-[11]. The performance assessment, carried out at CERN, has been a part of the acceptance criteria of the magnets. The test consisted in cooling down each magnet at operational temperature $(1.9 \mathrm{~K})$, and powering it up a current ranging between $11.85 \mathrm{kA}(7 \mathrm{TeV})$ and $12.85 \mathrm{kA}(7.7 \mathrm{TeV})$. The acceptance criterion at the beginning of the production was to reach $12 \mathrm{kA}$ after 2 quenches. In case of not fulfilling this first criterion, the magnet had to reach $12.85 \mathrm{kA}$ within 8 quenches [9], [11]. When the production speeded up, the first criterion was changed to reaching $12.25 \mathrm{kA}$ after 3 quenches. In case of not reaching $12.85 \mathrm{kA}$ after 8 quenches, the magnet was warmed up and cooled down, to perform a second test [9]-[11].

\section{B. Data Representation}

In the literature, quench data of accelerator magnets have been usually represented as training curves of one or more magnets (see Figs. 1 and 2), or as an histogram of the first (or second) quench over a family of magnets [12]. During the LHC production, data have been often presented as histograms giving the number of quenches needed to reach nominal or ultimate current [9], [10].

On the other hand, during commissioning or operation, current is fed in a circuit where 154 magnets are in series, and one records the quench of a string of magnets. This led us to propose a plot where the data of individual test are gathered as if these magnets were tested in a string [13]. One builds a curve giving the total number of quenches $n(I)$ observed in a string of magnets to reach a given level of current $I$ : quenches of all magnets are put together and ordered by the current level, neglecting whether the quench is the first one, the second one, or the third one for that magnet. Data of Fig. 3 correspond to the hypothetical result of the training of a string containing the first 9 LHC dipoles made by Firm1. One remarks a much smoother curve then in Fig. 2.

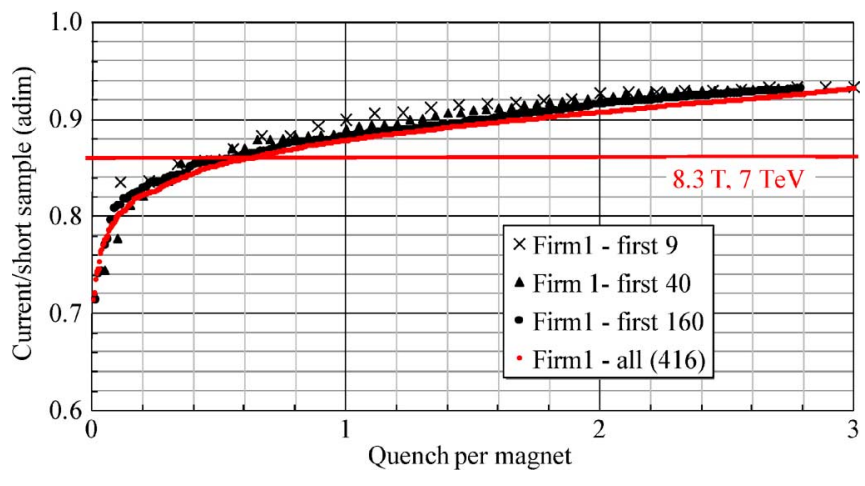

Fig. 4. Training curve for all the LHC dipoles of Firm1, virgin conditions, according to test data.

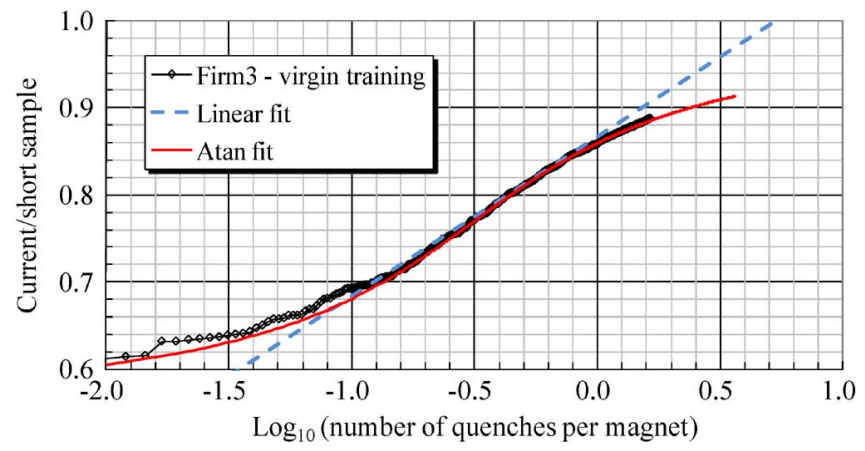

Fig. 5. Training curve for all the LHC dipoles of Firm3, virgin conditions, and empirical fits.

The successive step is to normalize the quenches to the number of magnets, and to normalize the current to the short sample current. The remarkable feature of this plot is that the quench behavior of a large string of magnets looks (i) very smooth (see Fig. 4) compared to the original individual data and (ii) for a stable production, it is independent of the number of magnets in the string. This second feature is not always observed: some batches can be less homogeneous.

\section{Exponential Fits of Training Curves}

When, during the hardware commissioning, the first sector of LHC dipoles was trained to push it toward nominal current, it was observed [14] that the level of current reached versus the number of quenches followed a semi-logarithmic behavior. To our knowledge, this simple empirical fit has been proposed for the first time on the basis of the LHC hardware commissioning data. Indeed, the behavior was clearly visible also in the test data, see Fig. 5 for the virgin data of Firm3. The fit reads

$$
n(I)=\exp \frac{I-I_{0}}{I_{1}}, \quad \text { or } \quad I(n)=I_{0}+I_{1} \log n
$$

where $n(I)$ is the number of quenches needed to reach the current $I$ in a string of $N$ magnets.

The fit is unphysical, since for $n \rightarrow \infty$ the magnet string reaches infinite currents. To get a more physical fit, one can consider the linear behavior as the central part of an arctangent function, having its asymptote at short sample current $I=I_{s s}$. This function is also suggested by the empirical observation that for 


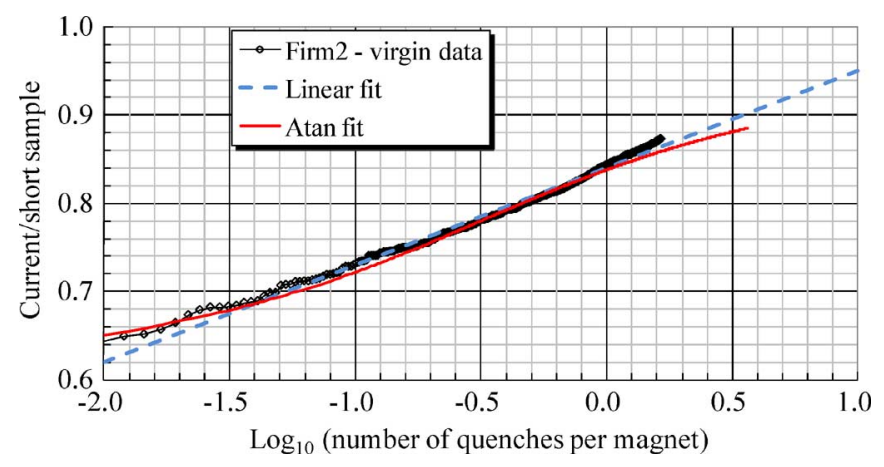

Fig. 6. Training curve for all the LHC dipoles of Firm2, virgin conditions, and empirical fits.

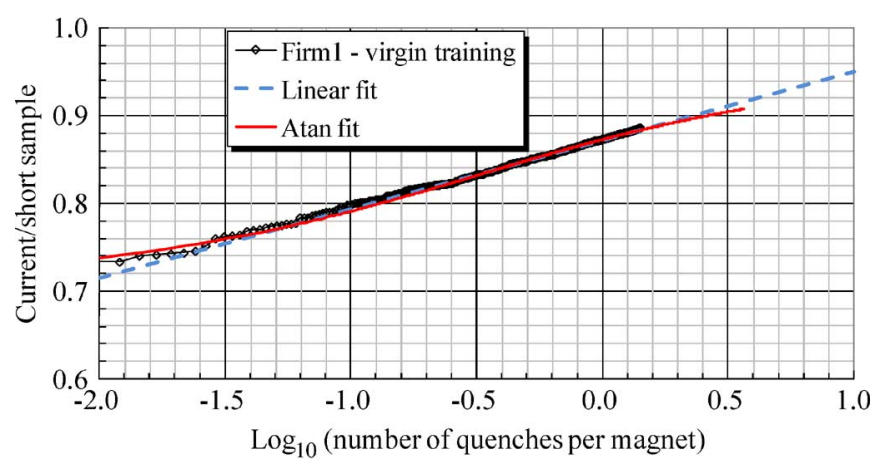

Fig. 7. Training curve for all the LHC dipoles of Firm1, virgin conditions, and empirical fits.

few quenches the linear approximation also gives an underestimate of the current reached. To quote Von Neumann [15], "With four parameters I can fit an elephant, and with five I can make him wiggle his trunk". Here we have just three parameters, so we are still far from the elephant syndrome. The fit reads

$$
I(n)=I_{0}+\frac{2\left(I_{s s}-I_{0}\right)}{\pi} \arctan \left[s\left(\log n-\log n_{0}\right)\right]
$$

where $I_{0}$ is the quench current at the flex, $n_{0}$ is the position of the flex, and $s$ is related to the slope at the curve flex. According to the fit, one cannot have quenches below $2_{I 0}-I_{s s}$. This fit makes the simplifying hypothesis that the magnet has no degradation, i.e. it reaches asymptotically the short sample limit (see Figs. 5-7).

\section{Behavior of Virgin Magnets}

In Fig. 8 we summarize the global performance of the LHC magnets on the test bench during the first powering (virgin training). Data are split per manufacturer, and show interesting differences. The very first quench of Firm 3 and Firm2 are at $\sim 8-9 \mathrm{kA}$, and at $10 \mathrm{kA}$ for Firm1. After an average of 2 quenches, the magnets of each manufacturer reach very similar current values, ranging from 12.2 to $12.5 \mathrm{kA}$. This is not surprising since the performance limit is set by the design and not by the manufacturing conditions.

Up to $10 \mathrm{kA}$ the training is dominated by Firm 3 (two thirds) and Firm 2 magnets (one third). At $11 \mathrm{kA}$ half of the magnets of Firm 2 and of Firm 3 quenched, and only $10 \%$ of the Firm 1 magnets. To reach nominal one needs 0.6 quenches per magnet

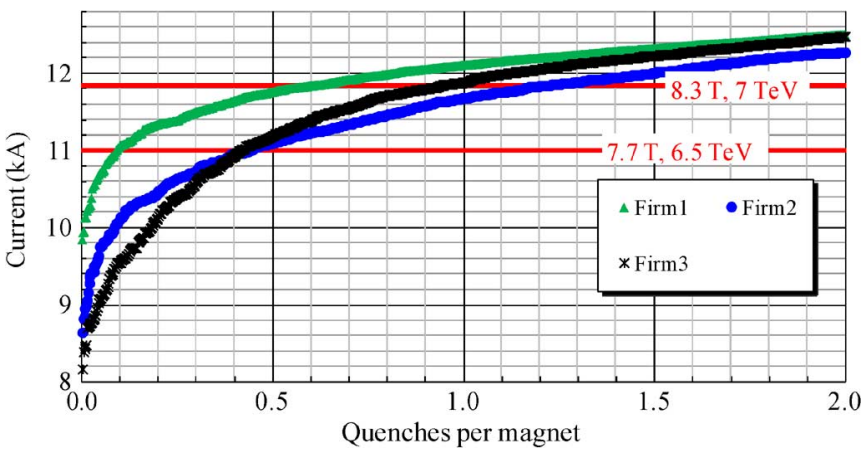

Fig. 8. Training curve for all the LHC dipoles, virgin conditions.

(Firm1), 1 quench per magnet (Firm3), and 1.3 quenches per magnet (Firm2).

The Firm 3 magnets have the worst quench behavior at low currents $(8-11 \mathrm{kA})$, but at the same time are the first ones to reach the top performance of $12.5 \mathrm{kA}$. Since during the production the main focus was not on the first level of quench, but rather on reaching the ultimate current, this is why Firm 3 magnets received the largest fraction of the bonus attributed for good performance. It also explains why, in the collective memory of our community, Firm 3 magnets have been always considered as having a good quench performance.

\section{E. Behavior After Thermal Cycle}

If the magnet did not satisfy the required quench performance during the first ("virgin") powering, it was warmed up and cooled down again for a second test campaign ("test after thermal cycle"). We have data relative to 136 magnets that were submitted to this treatment. These include not only the magnets with a bad performance in virgin conditions, but also a few magnets at the beginning of the production, when the testing time was not a bottleneck. This data sample clearly shows three features (see Fig. 9):

- If the first virgin quench is very low there is a large gain after thermal cycle, whereas if it is high there is a small gain: a magnet having a virgin quench at $9 \mathrm{kA}$ will gain on average $3 \mathrm{kA}$, i.e., it will start at $12 \mathrm{kA}$ after a thermal cycle. On the other hand, magnets starting at $11 \mathrm{kA}$ will gain on average only $1 \mathrm{kA}$ after a thermal cycle.

- The random part of the correlation is large.

- For Firm1-2, the difference between the first quench after thermal cycle and the first virgin quench is always positive, i.e. a thermal cycle never deteriorates the performance. On the other hand, this difference is negative (up to $1 \mathrm{kA}$ ) for four Firm 3 magnets. This shows that Firm 3 magnets have a worse training retention. This adds up to the slower virgin training shown in Fig. 8.

\section{Quench Performance During Hardware COMMISSIONING}

In May 2008, sector 5-6 was trained during hardware commissioning. The first training quench appeared at around $10 \mathrm{kA}$, as in other sectors. After a rather fast gain of $500 \mathrm{~A}$ in a few quenches, the training slope went down, eventually reaching 


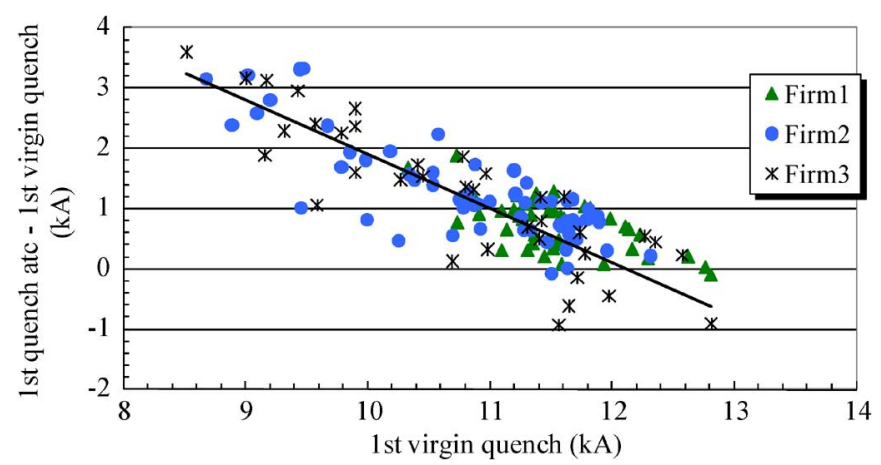

Fig. 9. Gain between the first quench after thermal cycle and the first virgin quench (markers), and linear fit for each firm (solid lines).

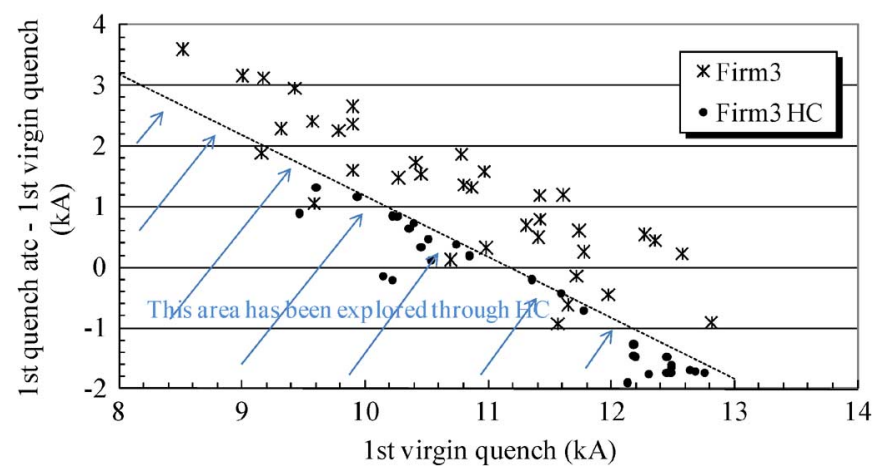

Fig. 10. Firm 3 data of the previous figure compared with the gain between quenches in the machine and 1st virgin quench.

$11.17 \mathrm{kA}$ after 28 'main' quenches. Among these 28 quenches, 26 were in Firm 3 magnets and 2 in Firm 2 magnets. Among the 26 Firm 3 quenches, in two cases two magnets were quenching at the same time (within $100 \mathrm{~ms}$ ) although spread apart by more than a hundred meters. Only one magnet, identified by the series number 3338, quenched twice. The successive quenches were always at higher current, with only one exception, i.e., after the 11th quench at $10.91 \mathrm{kA}$, and the 12 th at $10.72 \mathrm{kA}$. During the training, neighboring magnets underwent induced quenches typically at current of $1-7 \mathrm{kA}$.

The gain (or loss) in current between the first quench in the tunnel and the first virgin quench is shown in Fig. 10, where it is compared to the result relative to the sample of Firm 3 magnets tested after a thermal cycle. The dotted line indicates the region that has been explored during the training campaign of 5-6. One clearly sees an anomalous number of magnets with worse training retention.

\section{BEHAVIOR AFTER A THERMAL CYCLE}

\section{A. Monte-Carlo Based on Correlations Between Virgin Quenches and Quenches After a Thermal Cycle}

We used the following method to simulate the training of the LHC magnets of sector 5-6: (i) for each magnet we start from the first quench in virgin conditions on the test bench. Then, (ii) we apply the correlation (one per Firm) established in the previous section (see Fig. 9) to estimate the first quench after thermal cycle, made up of a deterministic part plus a stochastic one modeled through a Gaussian distribution worked

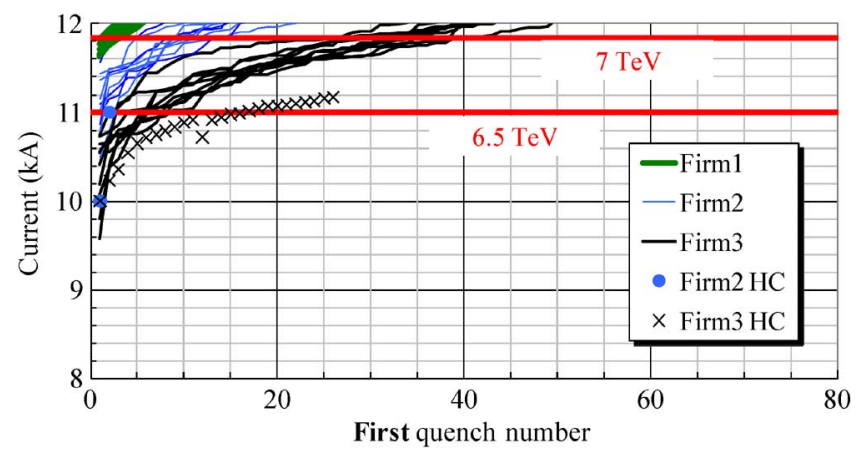

Fig. 11. Monte-Carlo to simulate the training of sector 5-6 in the tunnel (lines) and hardware commissining (HC) results (markers).

TABLE I

ESTIMATES OF THE QUENCHES NEEDED TO REACH NOMINAL IN SECTORS 5-6 AND IN THE LHC ACCORDING TO THE MONTE-CARLO METHOD

\begin{tabular}{|c|c|c|c|c|c|c|}
\hline & \multicolumn{2}{|c|}{ Sector 5-6 } & \multicolumn{2}{|c|}{ A generic octant } & \multicolumn{2}{|c|}{ All the LHC } \\
\hline & $\begin{array}{c}\% \text { of } \\
\text { magnets }\end{array}$ & $\begin{array}{c}\text { n. of } \\
\text { quenches }\end{array}$ & $\begin{array}{c}\% \text { of } \\
\text { magnets }\end{array}$ & $\begin{array}{c}\text { n. of } \\
\text { quenches }\end{array}$ & $\begin{array}{l}\% \text { of } \\
\text { magnets }\end{array}$ & $\begin{array}{c}\text { n. of } \\
\text { quenches }\end{array}$ \\
\hline Firm1 & $19 \%$ & 5 & $33 \%$ & 9 & $33 \%$ & 72 \\
\hline Firm2 & $26 \%$ & 15 & $33 \%$ & 19 & $33 \%$ & 155 \\
\hline Firm3 & $56 \%$ & 35 & $33 \%$ & 21 & $33 \%$ & 168 \\
\hline Total & $100 \%$ & 55 & $100 \%$ & 49 & $100 \%$ & 395 \\
\hline
\end{tabular}

out from the data shown in Fig. 9. Non Gaussian distributions more adapted to the data gave similar results. This foresees the first quench for each magnet. Second and third quenches could be added through building additional correlation plots between the first virgin quench and the second quench after thermal cycles, and so on. Since the hardware commissioning showed that nearly all magnets quench only once, this is neglected.

We repeat the procedure 10 times: results are shown in Fig. 11. The simulation for sector 5-6 shows that first quenches should appear at $10 \mathrm{kA}$, and that up to $11 \mathrm{kA}$ the quenches should occur in Firm 3 magnets, plus a few Firm 2 magnets. This is in agreement with the commissioning data. Indeed, the analysis shows that after 27 quenches the sector reaches $11.2 \mathrm{kA}$ instead of $11.6-11.8 \mathrm{kA}$.

The above method gives about 35 quenches for Firm 3 magnets, 15 for Firm 2 magnets, and 5 for Firm 1 magnets to reach nominal in sector 5-6, i.e. a total of 55 quenches (see Table I).

In fact 5-6 is a special case as it contains $55 \%$ of Firm 3 magnets. For a generic sector with one third of dipoles per Firm, one would have a total number of 50 quenches, giving 400 quenches over the whole LHC to reach nominal. This number is considerably larger than that given in [9]. In the following we analyse the reasons for this discrepancy.

\section{B. Scaling}

Estimates of the needed training in the tunnel have been given in [9] using a sample, and based on a linear scaling: (i) 1232 magnets took in virgin conditions about 1 quench per magnet to reach nominal, (ii) in the 136 magnets tested after thermal cycle, one observes a $80 \%$ reduction of the number of quenches needed to go to nominal after thermal cycle (241 quenches needed in virgin conditions, 48 after the thermal cycle); (iii) applying the $80 \%$ reduction to the 1200 quenches needed for the whole LHC, 


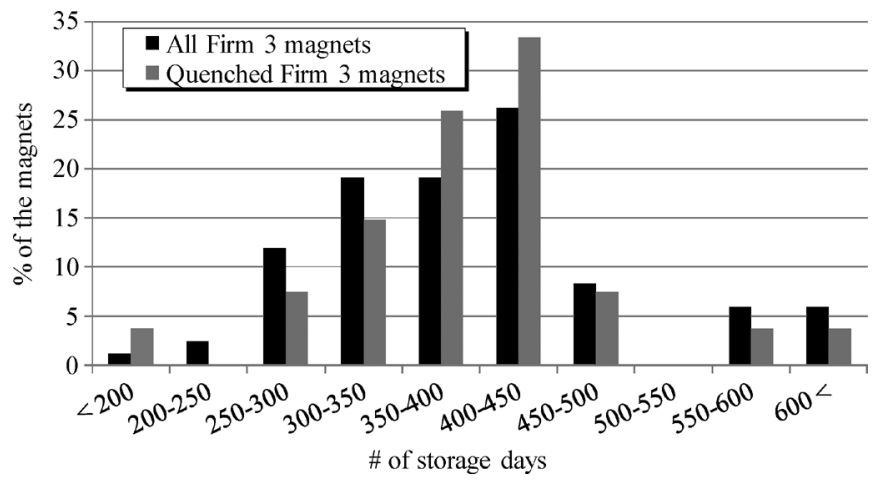

Fig. 12. Distribution of storage time for Firm 3 magnets in 5-6, and for the subset which quenched.

one gets 240 quenches, i.e. about 30 quenches per octant. This is much lower than the 50 quenches estimated with the MonteCarlo approach.

The apparent incoherency is subtle: the reason is that the sample is biased, since the magnets tested after the thermal cycle are the 'bad' ones, i.e., had a long virgin training. This is clearly seen from the number of quenches needed to reach nominal: $240 / 138=1.75$ quenches per magnet instead of 1 . If we make the extreme hypothesis that the behavior before and after the thermal cycle is totally uncorrelated, we see that the reduction factor of $80 \%$ has been overestimated, since we chose a biased sample having a twice longer virgin training.

On the other hand, assuming that all the LHC magnets behave in the tunnel as the sample of magnets tested after thermal cycle, we will have $48 / 138=0.35$ quenches per magnet to reach nominal, i.e. about 55 quenches per octant. This is much closer to the Monte Carlo estimate given in Table I.

\section{Extrapolation Based on Fits of Hardware Commissioning}

An alternative method is a simple extrapolation of the linear fit on a semilog scale of the 5-6 hardware commissioning data [13]. This method gives $900 \pm 300$ quenches for the whole LHC. Using the arctangent fit one obtains 600 quenches.

\section{Storage Time}

Some magnets of the LHC have been stored for a long time (up to two years). One of the first hypotheses to explain the worse training retention of Firm 3 magnets is that the long-term storage could have affected the performance. The analysis of sector 5-6 magnets shows that there is no indication of such a phenomenon (see Fig. 12).

\section{CONCLUSIONS}

Thanks to the very large statistics of LHC main dipole quench performance we showed that, (i) while the quench behavior of individual magnets is relatively random, order appears when the quench of a string of magnets is considered: an invariant training curve emerges. This curve is a powerful tool to characterize the performance of the magnet system, and, when normalized also to the short sample current, allows to compare the quench performance and the training between different families of magnets. Moreover, (ii) this curve has a simple expression: the number of quenches to reach a given current is an exponential of the current (see (1) and Figs. 2-6). This empirical two parameter fit can be made more physical by adding a third parameter (see (2)).

During the 2008 LHC commissioning, the whole set of 1232 LHC dipoles reached $9 \mathrm{kA}$, i.e. $75 \%$ of the nominal field, without a quench. One of the LHC sectors, i.e. 154 magnets, has been trained towards nominal, reaching $11.17 \mathrm{kA}(95 \%$ of nominal, corresponding to $6.6 \mathrm{TeV}$ ) with $\sim 30$ quenches. Rather surprisingly, almost all the quenches occurred in the magnets made by Firm3. This behavior can be partially derived from the data collected during the 5-years-long intensive test campaign on individual magnets carried out during the production through a Monte-Carlo method (see Fig. 11). Whereas $6.5 \mathrm{TeV}$ seems at hand with a few quenches per octant, $7 \mathrm{TeV}$ could need an intensive quench campaign. Several hypotheses are being considered to explain this partial loss of training, but there is no satisfactory explanation. The training of other sectors will shed more light on this issue.

\section{ACKNOWLEDGMENT}

The authors thank all the colleagues involved in the LHC dipole manufacturing and testing, and T. Taylor for reading the manuscript and useful comments.

\section{REFERENCES}

[1] D. Overbye, "Giant particle collider struggles," New York Times, August 3, 2009

[2] M. N. Wilson, Superconducting Magnets. Oxford: Clarendon Press, 1983.

[3] K. Mess, P. Schmuser, and S. Wolff, Superconducting Accelerator Magnets. , Singapore: World Scientific, 1996.

[4] A. Devred, "Quench origins," in AIP Conference Proceedings 249, M. Month and M. Dienes, Eds., 1992, pp. 1309-1372.

[5] H. Brechna et al., "Superconducting magnets for high energy accelerators," IEEE Trans. Magn., vol. 17, p. 2355, 1981.

[6] A. Tollestrup, "Care and training of superconducting magnets," IEEE Trans. Magn., vol. 17, p. 863, 1981.

[7] A. Siemko et al., these proceedings.

[8] A. Verweij et al., "Performance of the main dipole magnet circuits of the LHC during commissioning," in EPAC, 2008, p. 2473.

[9] P. Pugnat and A. Siemko, "Review of quench performance of LHC main magnets," IEEE Trans. Appl. Supercond., vol. 17, p. 1091, 2007.

[10] A. Siemko and P. Pugnat, "Performance evaluation and quality assurance management during the series power tests of LHC main lattice magnets," IEEE Trans. Appl. Supercond., vol. 18, p. 126, 2008.

[11] V. Chohan et al., "LHC magnet tests: Operational techniques and empowerment for successful completion," in PAC, 2007.

[12] R. Meinke, "Superconducting magnet system for HERA," IEEE Trans. Appl. Supercond., vol. 27, p. 1728, 1991.

[13] B. Bellesia, N. C. Lasheras, and E. Todesco, "Magnet (re)training," in Chamonix Workshop, 2009.

[14] A. Verweij, "Training the dipoles," in Chamonix Workshop, 2009.

[15] F. Dyson, "A meeting with Enrico Fermi," Nature, vol. 427, p. 297, 2004. 